\title{
YAP activates the Hippo pathway in a negative feedback loop
}

Cell Research (2015) 25:1175-1178. doi:10.1038/cr.2015.101; published online 28 August 2015

\section{Dear Editor,}

A relatively constant organ size is one of the most striking yet mysterious features of multicellular organisms, which was recently found to be regulated by the Hippo pathway in an evolutionarily conserved manner. Inactivation of this pathway leads to drastic enlargement of organ size due to a marked increase of cell number [1]. Furthermore, deregulation of the Hippo pathway plays broad roles in human cancer development from tumorigenesis to cancer relapse [2]. Mechanistically, Yes-associated protein (YAP), a transcription co-activator and a potent growth promoter, is directly phosphorylated by the Hippo pathway kinases Lats1 and Lats2, and then inhibited through cytoplasmic retention and degradation. Chemical and physical signals such as GPCR ligands and matrix stiffness dampen the Hippo pathway, thus leading to YAP activation, which facilitates cell proliferation, evasion from apoptosis, and stem cell self-renewal [3]. Therefore, the amplitude and duration of YAP activation in response to physiological stimulations should be precisely controlled to achieve proper development and tissue homeostasis. Here we report that YAP activation strongly activates its upstream inhibitory kinases Lats $1 / 2$ partially via directly inducing transcription of Lats 2 and AMOTL2 and possibly further via increasing the protein level of NF2, the Hippo pathway upstream component. Our data suggest that YAP activity is limited by a negative feedback mechanism, which serves to cancel signal noise during development and thus ensure tissue homeostasis.

Genetic and biochemical studies have demonstrated that phosphorylation of YAP by Lats $1 / 2$ on five serine sites is the key mechanism limiting YAP activity [4]. Mutation of all five serines to alanines (5SA) generates a highly tumorigenic, constitutively active YAP mutant resistant to inhibition by the Hippo pathway. The YAP5SA mutant is thus widely used as a mimic of Hippo pathway inactivation. Surprisingly, we found that expression of YAP-5SA markedly induced phosphorylation of endogenous YAP as indicated by a strong upshift on the
Phos-tag (PT) gel, which was further confirmed by an antibody against YAP phosphorylated at S127 (the major phosphorylation site regulated by the Hippo pathway; Figure 1A). Furthermore, when YAP-5SA was expressed in several different cell lines, Lats $1 / 2$ phosphorylation on the hydrophobic motif was drastically enhanced, suggesting their activation (Figure 1A, 1C and Supplementary information, Figure S1A). Indeed, in vitro kinase assay demonstrated that endogenous Lats 1 and Lats2 from YAP-5SA-expressing cells were highly activated (Figure 1B). Consistently, phosphorylation of Angiomotin (AMOT), another functional target of Lats $1 / 2[5,6]$, was also strongly induced in YAP-5SA-expressing cells with an elevated protein level possibly due to protein stabilization (mRNA level not elevated, data not shown; Figure 1A). The above data clearly indicate that YAP activation unexpectedly activates its upstream inhibitory kinases Lats $1 / 2$, likely forming a negative feedback mechanism.

By examining the expression of Hippo pathway proteins we found that Lats2 protein level was consistently elevated by YAP activation across cell lines while Lats 1 and NF2 protein levels were elevated in a cell type-dependent manner (Figure 1A, 1C and Supplementary information, Figure S1A). In contrast, Mst1/2 and Sav protein levels remain similar between control and YAP-5SA-expression cells (Figure 1A). We further examined whether the elevation of Lats $1 / 2$ phosphorylation and Lats2 protein levels depends on YAP transcriptional activity. YAP serine 94 (S94) is essential for its interaction with TEADs, major transcription factors mediating YAP functions [7]. S94A mutation largely abolished YAP-5SA-induced increase in Lats1/2 phosphorylation and Lats2 protein levels (Figure 1C). Furthermore, deletion of the C-terminal YAP transcription activation domain also abolished YAP-5SA-induced Lats $1 / 2$ activation (Figure 1C). Therefore, YAP-induced negative feedback is dependent on YAP-TEAD transcriptional activity. We next asked whether the elevation of Lats2 and sometimes Lats1 and NF2 protein levels induced by YAP activation is due to their enhanced transcription. We found that Lats2 but not Lats1 or NF2 transcription 


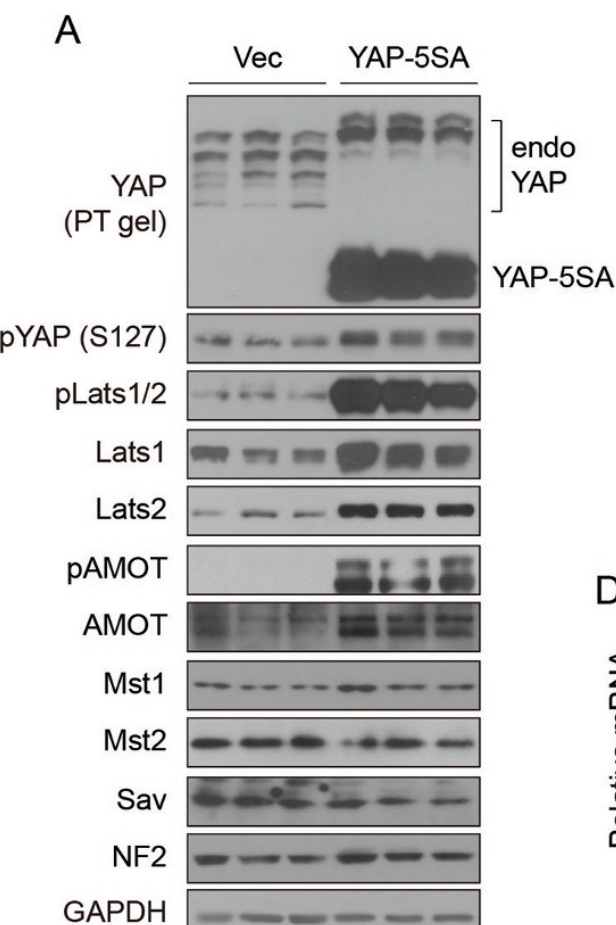

$\mathrm{F}$

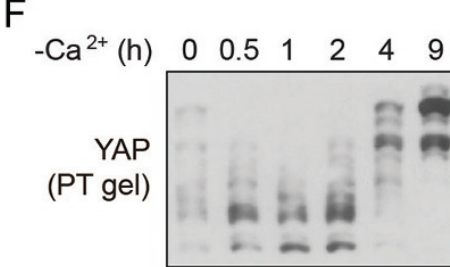

pLats $1 / 2$

Lats

Lats2

NF2

Tubulin

G

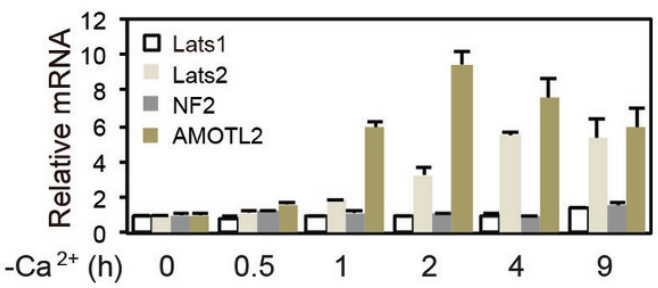

B

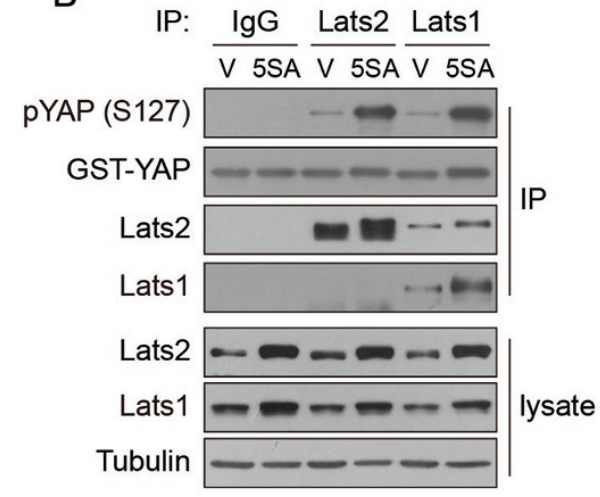

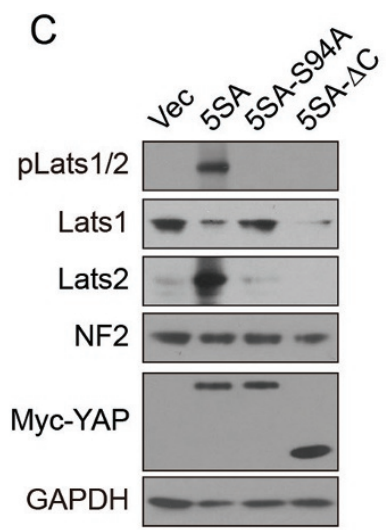

D

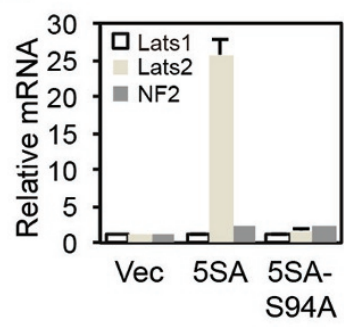

E

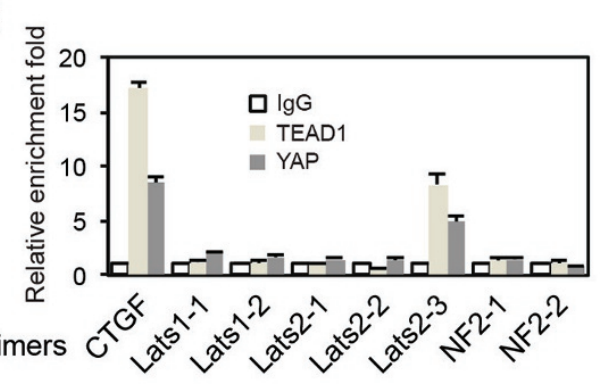

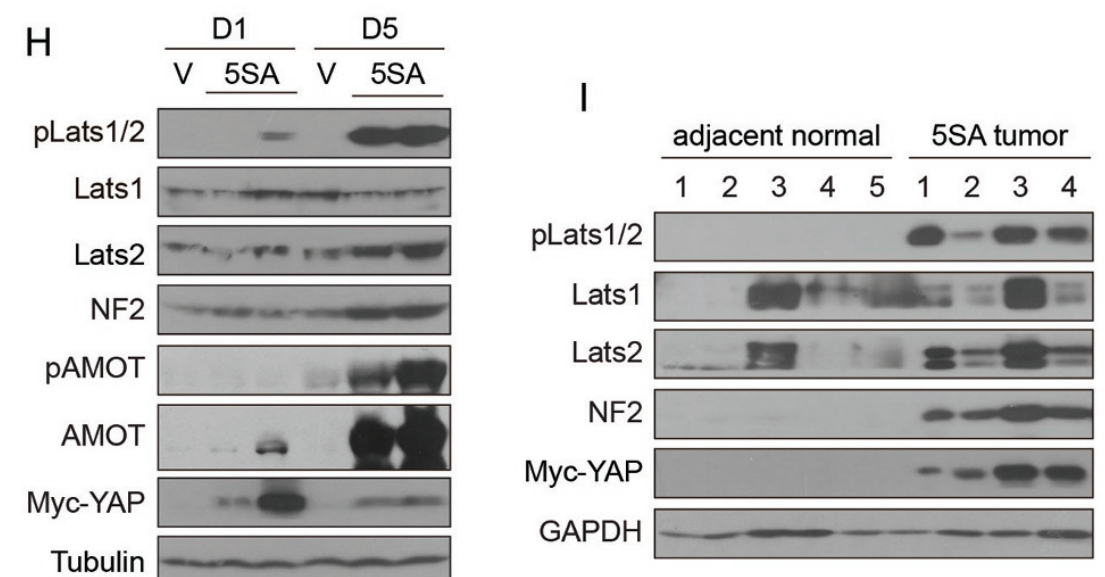

$\mathrm{J}$

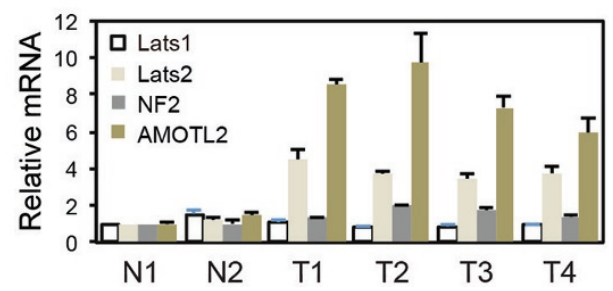

was induced by YAP activation to different extents across cell lines (Figure 1D and Supplementary information, Figure S1B). In contrast, expression of active YAP could not block Lats $1 / 2$ degradation upon inhibition of protein synthesis, although at an intermediate time point Lats2 degradation seems to be slower possibly due to a much higher basal Lats2 protein level (Supplementary information, Figure S1C). Thus alteration of Lats2 stability is unlikely to be the major reason for elevated Lats2 protein level induced by YAP activation. Furthermore, chromatin 
Figure 1 YAP activates the Hippo pathway in a negative feedback loop. (A) Constitutively active YAP strongly induces Lats $1 / 2$ phosphorylation. Control and YAP-5SA-expressing HMLE cells were lysed and the lysates were analyzed by western blot analysis. Results of three experiments are shown. (B) Constitutively active YAP activates Lats $1 / 2$ kinases. Endogenous Lats 1 and Lats2 were immunoprecipitated from control and YAP-5SA-expressing HMLE cells and subjected to in vitro kinase assay using GST-YAP as the substrate. Detection of Lats2 in Lats1 immunoprecipitates is either due to interaction between Lats1 and 2 or cross-recognition by the antibody. (C) Activation of Lats $1 / 2$ by YAP depends on the TEAD-binding ability and the transcriptional activity of YAP. MCF10A cells expressing the indicated YAP mutants were lysed and the lysates were analyzed by western blot analysis. (D) YAP induces Lats2 transcription in a TEAD-dependent manner. mRNA was extracted from the indicated MCF10A stable cells. The mRNA levels of Lats1, Lats2, and NF2 were analyzed by quantitative RT-PCR. (E) YAP and TEAD1 bind to Lats2 promoter. HepG2 cells were processed for ChIP experiments with control IgG or antibodies against TEAD1 or YAP followed by quantitative PCR. CTGF was used as a positive control. (F) YAP activation upon loss of cell-cell junctions activates Lats1/2. Confluent MDCK cells were switched to calcium-free culture medium for the indicated time. Cell lysates were examined by western blot analysis. (G) YAP activation upon loss of cell-cell junctions induces Lats2 and AMOTL2 transcription. Cells were treated in the same way as described in F. mRNA was extracted and analyzed by quantitative RT-PCR. (H) YAP activation in mouse liver activates Lats $1 / 2$. Mice were hydrodynamically injected with empty vector or YAP-5SA transposon plasmids with PB transposase plasmids. On post-injection day 1 and day 5 , livers were harvested and lysed, and the lysates were analyzed by western blot analysis. (I) Lats1/2 are constantly activated in YAP5SA-induced tumors. Tumors and adjacent normal tissues (4 and 5 samples, respectively, as indicated) were dissected from YAP5SA-hydrodynamically injected mice 100 days after the injection. Tissues were lysed and the lysates were analyzed by western blot analysis. (J) Lats2 and AMOTL2 but not NF2 mRNA levels were elevated in YAP-5SA-induced tumors. mRNA was extracted from tissues described in I. mRNA levels of Lats1, Lats2, NF2, and AMOTL2 were analyzed by quantitative RT-PCR.

immunoprecipitation (ChIP) experiments showed that TEAD1 and YAP directly bind to the proximal region of the Lats 2 promoter, where potential TEAD-binding elements could be spotted, but not to other regions of the Lats 2 promoter or Lats 1 and NF2 promoters (Figure 1E). These findings demonstrate that YAP directly induces Lats 2 transcription through TEADs, which partially contributes to a feedback activation of the Hippo pathway. The observed alteration of Lats1 protein levels (Figure 1A) could be partially due to cross-recognition of Lats2 by Lats 1 antibody as could be seen in Figure 1B.

We next asked whether such a YAP-induced negative feedback exists upon endogenous YAP activation by physiological signals. The Hippo pathway is known to be regulated by physical signals such as cell-cell contact and chemical signals such as lysophosphatidic acid in serum $[8,9]$. We found that upon disruption of cell-cell junctions following calcium removal from the culture medium, YAP was quickly dephosphorylated and activated (Figure 1F). However, after YAP activation, YAP phosphorylation gradually recovered from $4 \mathrm{~h}$ post the treatment, which was accompanied by a steady increase of Lats $1 / 2$ phosphorylation and Lats 2 mRNA and protein levels (Figure $1 \mathrm{~F}$ and $1 \mathrm{G}$ ). Interestingly, we found that the mRNA level of AMOTL2, which was shown to be an activator of Lats1/2 [10], was also quickly elevated following YAP activation (Figure 1G). The induction of AMOTL2 may further explain the feedback activation of Lats $1 / 2$ upon YAP activation. Indeed, AMOTL2 expression level is also elevated in YAP-5SA-expressing cells (Supplementary information, Figure S1D). Moreover, ChIP experiments showed that AMOTL2 is also a direct target gene of YAP and TEADs (Supplementary information, Figure S1E). In the context of serum-induced YAP activation, we also observed gradual increase of Lats2 and AMOTL2 expression and Lats $1 / 2$ phosphorylation levels followed by a dramatic enhancement of YAP phosphorylation (Supplementary information, Figure S1F and S1G). These data support that physiological activation of YAP is followed by a feedback activation of Lats 2 transcription and Lats1/2 kinase activity, which normalizes YAP activity.

Disruption of the Hippo pathway or ectopic expression of active YAP in mouse liver leads to tumorigenesis [2]. However, as demonstrated above, upon YAP activation, Lats $1 / 2$ could be activated in an attempt to normalize YAP activity. We examined this possibility in vivo by expressing YAP-5SA transposon plasmids in mouse liver using hydrodynamic injection. Remarkably, while Lats $1 / 2$ phosphorylation was only mildly induced on post-injection day 1 , we observed strong Lats $1 / 2$ phosphorylation on day 5 , which was accompanied by elevated Lats2 and NF2 protein levels (Figure 1H). Marked increase of AMOT phosphorylation and protein levels further confirmed activation of Last $1 / 2$ kinase activity (Figure 1H). Thus, YAP activation indeed induces a negative feedback loop in vivo. However, as YAP-5SA is insensitive to the Hippo pathway kinases, tumorigenesis occurred within 3 months regardless of Hippo pathway activation. Comparing dissected tumors with adjacent normal tissues we found that Lats $1 / 2$ were still activated with further enhanced protein level of NF2 (Figure 1I). However, Lats2 and AMOTL2 but not NF2 mRNA levels were elevated in tumors (Figure 1J). Furthermore, we observed that YAP activation upon liver-specific Mst1/2 double knockout (DKO) was accompanied by increased Lats2 and AMOTL2 mRNA and NF2 protein levels (Supplementary information, Figure S1H and S1I). These 
data suggest that YAP-induced Lats2 and AMOTL2 transcription is responsible for sustained Lats $1 / 2$ activation, and that NF2 protein level is elevated by YAP activation in vivo through additional mechanisms.

Activation-initiated negative feedback is a common principle in signal transduction. For instance, activation of EGFR induces multiple layers of negative feedback regulation such as recruitment of phosphatases, receptor endocytosis and degradation, and transcription-based induction of negative regulators [11]. Installation of negative feedback mechanisms partially serves to dampen noise and prevent signal fluctuation. YAP, the major effector of the Hippo pathway, on one hand holds extremely potent growth-promoting activity and on the other is modulated by complex chemical and physical stimuli. Thus, precise regulation of YAP activity is crucial to ensure proper development and to prevent abnormal growth that leads to tumorigenesis. Although Drosophila Yki was shown to induce transcription of Hippo pathway components ex, merlin, four-jointed, and kibra [1], a negative feedback mechanism of YAP was previously unknown. In this report we found that YAP and TEAD directly bind to Lats2 and AMOTL2 promoters and activate their expression. Upon YAP activation in vitro and in vivo by expressing constitutively active YAP or by physiological stimuli, Lats2 and AMOTL2 expression was induced, contributing to a negative feedback loop. Elevation of NF2 protein level is also likely involved in the feedback especially after prolonged YAP activation in vivo. Our data do not exclude the possibility of additional mechanisms underlying the feedback inactivation of YAP. Furthermore, it is also likely that activation of YAP would lead to phosphorylation of other Lats $1 / 2$ kinase substrates, which may contribute to the biological functions of the Hippo pathway.

In conclusion, our study reveals that activation of YAP strongly activates its upstream inhibitors Lats $1 / 2$ kinases, thus establishing a previously unknown negative feedback mechanism of the Hippo pathway. This mechanism likely plays an important role in buffering the noise of YAP-activating signals to guarantee proper tissue development and to prevent tumorigenesis. While this manu- script was in preparation, two independent studies reported similar findings that YAP induces a negative feedback loop involving transcriptional induction of Lats2 and induction of NF2, which are consistent with our observations $[12,13]$.

\section{Acknowledgments}

We thank Drs Zengqiang Yuan and Yingzi Yang for Mst1/2-knockout mice, Dr Yong Cang for Albumin-Cre mice, and Drs Tian Xu and Xiaohui Wu for the piggyBac system. This work was supported by the National Natural Science Foundation of China (31271508, 31471316 and 31422036), the State Key Development Program for Basic Research of China (2013CB945303), Natural Science Foundation of Zhejiang (LR12C07001), Specialized Research Fund for the Doctoral Program of Higher Education (20130101110117), the 111 project (B13026), Fundamental Research Funds for Central Universities of China (2015XZZX004-17), and the Thousand Young Talents Plan of China.

\section{Xiaoming Dai ${ }^{1, *}$, Huan Liu ${ }^{1, *}$, Shuying Shen ${ }^{1,2}$, Xiaocan Guo ${ }^{1}$, Huan Yan $^{1}$, Xinyan $\mathrm{Ji}^{1}$, Li Li ${ }^{3}$, Jun Huang ${ }^{1}$, Xin-Hua Feng ${ }^{1}$, Bin Zhao ${ }^{1}$}

${ }^{I}$ Life Sciences Institute and Innovation Center for Cell Signaling Network, Hangzhou, Zhejiang 310058, China; ${ }^{2}$ Institute of Biochemistry, College of Life Science, Hangzhou, Zhejiang 310058, China; ${ }^{3}$ Institute of Aging Research, Hangzhou Normal University, Hangzhou, Zhejiang 311121, China

*These two authors contributed equally to this work.

Correspondence: Bin Zhao

E-mail: binzhao@zju.edu.cn

\section{References}

1 Pan D. Dev Cell 2010; 19:491-505.

2 Moroishi T, Hansen CG, Guan KL. Nat Rev Cancer 2015; 15:73-79.

3 Yu FX, Guan KL. Genes Dev 2013; 27:355-371.

4 Zhao B, Li L, Tumaneng K, et al. Genes Dev 2010; 24:72-85.

5 Dai X, She P, Chi F, et al. J Biol Chem 2013; 288:34041-34051.

6 Chan SW, Lim CJ, Guo F, et al. J Biol Chem 2013; 288:37296-37307.

7 Zhao B, Ye X, Yu J, et al. Genes Dev 2008; 22:1962-1971.

8 Zhao B, Wei X, Li W, et al. Genes Dev 2007; 21:2747-2761.

9 Yu FX, Zhao B, Panupinthu N, et al. Cell 2012; 150:780-791.

10 Paramasivam M, Sarkeshik A, Yates JR 3rd, et al. Mol Biol Cell 2011; 22:3725-3733.

11 Lemmon MA, Schlessinger J. Cell 2010; 141:1117-1134.

12 Chen Q, Zhang N, Xie R, et al. Genes Dev 2015; 29:1285-1297.

13 Moroishi T, Park HW, Qin B, et al. Genes Dev 2015; 29:1271-1284.

(Supplementary information is linked to the online version of the paper on the Cell Research website.) 